\title{
Higher Heart-Rate Variability Is Associated with Ventromedial Prefrontal Cortex Activity and Increased Resistance to Temptation in Dietary Self-Control Challenges
}

\author{
(I)Silvia U. Maier and ๑Todd A. Hare \\ Department of Economics, Laboratory for Social and Neural Systems Research, University of Zurich, CH-8006 Zurich, Switzerland
}

\begin{abstract}
Higher levels of self-control in decision making have been linked to better psychosocial and physical health. A similar link to health outcomes has been reported for heart-rate variability (HRV), a marker of physiological flexibility. Here, we sought to link these two, largely separate, research domains by testing the hypothesis that greater HRV would be associated with better dietary self-control in humans. Specifically, we examined whether total HRV at sedentary rest (measured as the SD of normal-to-normal intervals) can serve as a biomarker for the neurophysiological adaptability that putatively underlies self-controlled behavior. We found that HRV explained a significant portion of the individual variability in dietary self-control, with individuals having higher HRV being better able to downregulate their cravings in the face of taste temptations. Furthermore, HRV was associated with activity patterns in the ventromedial prefrontal cortex (vmPFC), a key node in the brain's valuation and decision circuitry. Specifically, individuals with higher HRV showed both higher overall vmPFC blood-oxygen-level-dependent activity and attenuated taste representations when presented with a dietary self-control challenge. Last, the behavioral and neural associations with HRV were consistent across both our stress induction and control experimental conditions. The stability of this association across experimental conditions suggests that HRV may serve as both a readily obtainable and robust biomarker for self-control ability across environmental contexts.
\end{abstract}

Key words: decision making; fMRI; HRV; self-regulation

Significance Statement

Self-control is associated with better health, but behavioral and psychometric self-control measures allow only indirect associations with health outcomes and may be distorted by reporting bias. We tested whether resting heart-rate variability (HRV), a physiological indicator of psychological and physical health, can predict individual differences in dietary self-control in humans. We found that higher HRV was associated with better self-control and improved predictions of choice behavior. Specifically, higher HRV was associated with more effective downregulation of taste temptations, and with a diminished neural representation of taste temptations during self-control challenges. Our results suggest that HRV may serve as an easily acquired, noninvasive, and low-cost biomarker for self-control ability.

\section{Introduction}

Self-regulation has been associated with a wide range of life outcomes, from educational achievement and socioeconomic status to mental and physical health (Mischel et al., 1989; Duckworth,

Received Sept. 7, 2016; revised 0ct. 31, 2016; accepted Nov. 13, 2016.

Author contributions: S.U.M. and T.A.H. designed research; S.U.M. performed research; S.U.M. and T.A.H. analyzed data; S.U.M. and T.A.H. wrote the paper.

This work was supported by Swiss National Science Foundation Grant 140277 and EU FP7 Grant 607310. The authors thank Gioia Biriukoff, Elia In-Albon, and Sam Leone for help with the data collection and documentation.

The authors declare no competing financial interests.

Correspondence should be addressed to Silvia U. Maier, University of Zurich, Department of Economics, Laboratory for Social and Neural Systems Research, Bluemlisalpstrasse 10, CH-8006 Zurich, Switzerland. E-mail: silvia.maier@econ.uzh.ch.

DOI:10.1523/JNEUROSCI.2815-16.2016

Copyright $\odot 2017$ the authors $\quad 0270-6474 / 17 / 370446-10 \$ 15.00 / 0$
2011; Moffitt et al., 2011). Therefore, accurate predictors of individuals' self-regulatory abilities are important tools in both basic scientific research as well as applied domains, including education and medicine. Self-regulation is generally assessed in specific domains by psychometric questionnaires or laboratory tasks. Unfortunately, participants can potentially distort these measurements by reporting socially desirable answers or behaving according to the presumed goals of the experimenter. Therefore, measures based on physiological readouts that are easy to obtain, domain independent, and robust to reporting biases could be important tools in the assessment of self-control.

One such readout is heart-rate variability (HRV). Measures of HRV have been linked to self-regulatory capacities and performance in the domain of emotion (Thayer and Lane, 2000, 2009), raising the question of whether they might serve as more general 
predictors of self-control. HRV is a well established physiological characteristic of all vertebrates (Grossman and Taylor, 2007): the timing between subsequent heartbeats oscillates on the order of milliseconds and no two neighboring beat pairs (RR intervals) are of exactly the same length (Camm et al., 1996). An animal's HRV is sensitive to both physical and mental strain (Porges and Raskin, 1969), and differences in resting HRV can distinguish between states of health and disease (Heni et al., 2014, 2015). High resting HRV has been associated with good physical (Masi et al., 2007; Brändle et al., 2015) and mental health (Thayer and Brosschot, 2005), while chronic decreases in HRV indicated disease states and slow recovery from stress (Weber et al., 2010; Stalder et al., 2011).

The polyvagal (Porges, 1995, 2001) and neurovisceral integration (Thayer and Lane, 2000, 2009) theories postulate a mechanistic link between HRV and self-regulation. Both associate CNS regulation of the cardiovascular system, which is necessary to prepare reactions to challenges in the environment, with adaptive behavior at a higher cognitive level. However, we note that it remains unclear to what degree the central versus peripheral nervous system influences HRV. Although we do not yet fully understand all of the physiological and cognitive factors driving HRV (Heathers, 2014), we build on previous proposals (Grossman and Taylor, 2007) and posit that HRV serves as a readout of an individual's allostatic capacities to integrate behavioral strategies and energy stores in response to demands in the environment.

Higher HRV has been linked to several cognitive processes that support self-regulation, including the following: (1) reallocation of attention (e.g., disengaging from stimuli that are not threatening in the current context), which may reduce allostatic load (McEwen and Wingfield, 2003); (2) persistence (Reynard et al., 2011); and (3) working memory (Gianaros et al., 2004; Hansen et al., 2004). In contrast, low HRV has been associated with disinhibition and dysregulated social conduct (Beauchaine, 2001, 2007). Furthermore, Daly et al. (2014) reported that higher trait self-control, measured using the self-report scale developed by Tangney et al. (2004), correlates with higher resting HRV. However, the links between HRV and self-control at the neural level remain unknown.

In this study, we used fMRI to investigate the relationship between dietary self-control and resting HRV. We hypothesized that better self-control should be associated with higher HRV, and that individual differences in HRV would be associated with neural processing within a self-control network, including the dorsolateral prefrontal cortex (dlPFC) and the ventromedial prefrontal cortex (vmPFC; Hare et al., 2009; Maier et al., 2015). We indeed found that higher resting HRV was associated with better dietary self-control. Furthermore, we found that HRV positively correlated with activity in the vmPFC when individuals faced self-control challenges, and that high-HRV individuals showed a decreased sensitivity to taste attributes in the vmPFC. These vmPFC findings suggest a neural mechanism for the downregulation of tempting taste attributes that may facilitate dietary self-control.

\section{Materials and Methods}

Participants

Fifty-one men participated in this study. The sample is the same as in the study by Maier et al. (2015), where we reported the effects of stress on behavioral and neural self-control processes, but no heart-rate analyses. We included only male participants to facilitate the collection and analysis of cortisol responses to stress in our previous work. Baseline heartrate data for two participants were lost due to recording failure. In the present report, we include the subset of participants for whom we have both heart-rate and fMRI data (22 control and 27 stress group participants). The Ethics Committee of the Canton of Zurich approved this study and all participants provided written informed consent on the study day. All participants were right-handed and had normal or corrected to normal vision. None of them reported any history of somatic or psychiatric disease, nor did they take any prescription medication. On average, participants in the sample had a blood pressure in the (high) normal range for their age group (mean + SD: systolic blood pressure, $130 \pm 14 \mathrm{mmHg}$; diastolic blood pressure, $77 \pm 9 \mathrm{mmHg}$ ).

Participants were excluded during the recruitment stage if they suffered from any allergies, food intolerances, or eating disorders. We also excluded individuals who followed a specific diet (e.g., eating vegetarian, vegan, gluten-free/lactose-free, etc.), or who did not report enjoying and regularly consuming snack foods (regularly was defined as $>2$ occasions per week). A final eligibility criterion was that participants had to report that they were trying to maintain a healthy lifestyle, including exercise and an overall balanced diet. Together, these criteria ensured that participants would face a meaningful self-control challenge in the dietarychoice task.

To ensure a homogeneous reaction of the hypothalamic-pituitaryadrenal axis in response to stress induction, participants were asked to abstain from drinking alcoholic or caffeinated beverages in the $18 \mathrm{~h}$ before the study, to not exercise in the $6 \mathrm{~h}$ before the study, and to come to the laboratory well rested. We only recruited nonsmokers who had no history of drug abuse. We asked participants to go to bed by midnight at the latest on the day before the study and to get a good night's sleep. We instructed participants to not take any medication that alters the blood flow (e.g., analgesics) in the $72 \mathrm{~h}$ before their appointment. To motivate the dietary choices, participants were instructed to eat a small meal (sandwich or salad with $\sim 450 \mathrm{kcal}$ ) $3 \mathrm{~h}$ before the study and consume nothing but water after that.

Allen et al. (2007) identified age, exercise habits, and obesity as potential confounding factors for heart-rate analyses. Our sample was relatively homogeneous with regard to these factors. The men were on average 21.2 years old (SD, \pm 2 years), had a normal body mass index (BMI; mean + SD: $22.7 \pm 2.1$ ), trained on average $1.6(\mathrm{SD}, \pm 1.4)$ times per week for building strength, and had completed an average of 1.9 (SD, \pm 1.3 ) cardio training sessions per week during the past 4 weeks before the study, resulting in a combined mean of $3.6(\mathrm{SD}, \pm 2.1$ ) weekly training sessions per participant. Other factors identified by Allen and colleagues, including smoking, gender, caffeine and alcohol intake, and circadian rhythm, were controlled for by our study exclusion criteria and design.

\section{Procedure}

In the 30-40 min preceding the resting HRV measurement, participants had rated 180 food items for health and taste to create tempting dietary choice pairs. After the heartbeat-interval measurement, a stress induction [Socially Evaluated Cold Pressor Test (SECPT)] or control procedure was administered. Assignment to the stress induction or control conditions was unknown to both the participant and the experimenter at the time of HRV measurement. However, participants knew they would be randomly assigned to one treatment or the other based on the information provided with the consent forms at the beginning of the study. The SECPT treatment elicited an acute stress response, as indicated by higher cortisol values in the Stress group (mean \pm SEM cortisol at maximum: Stress, $9.64 \pm 1.09 \mathrm{~nm} / \mathrm{l}$; Control, $6.6 \pm 0.67 \mathrm{~nm} / \mathrm{l})$, and higher reports of perceiving to be stressed than the Control group (on a visual analog scale from 0 to 100 with 0 "not at all stressed and 100 "extremely stressed": mean \pm SEM, Stress, $33 \pm 4 \%$; Control, $19 \pm 5 \%$ ). Details of the stress induction and behavioral task were reported by Maier et al. (2015). Here, we focus on the relationship between the baseline HRV parameter and dietary self-control success (SCS) and its neural correlates. However, given that the stress treatment is known to change dietary choices (Maier et al., 2015), we included it as a factor in all regression models.

Immediately after the stress induction, participants were scanned with blood-oxygen-level-dependent (BOLD) fMRI while they made choices 
in a dietary self-control task. The screen always depicted two food items, and participants had to choose whether they wanted to eat the item on the right or on the left (Fig. 1a). The binary food choices fell into one of two categories. In the first, choosing the healthier item is trivial because the healthier item was also tastier. We refer to this category as no-challenge trials because there is no self-control challenge. In the second choice category, self-control challenges, the healthier item was the less tasty of the two foods and thus presented a conflict between taste and health attributes. Self-control challenges were presented on approximately half of the 210 trials for each participant. To examine the variability of the challenge that different participants faced, we normalized the ratings to fall between 0 and 100 points, and calculated absolute taste and health differences between every pair of food items each participant faced. On a scale from 0 to 100 , the mean health difference was $34 \pm 25$ points (mean \pm SD) and the mean taste difference was $29 \pm 21$ points.

The behavioral and fMRI analyses in this paper focus on the selfcontrol challenge cases. Before the task started, participants were reminded to choose the healthier item as often as they could, consistent with their healthy lifestyle goals. Participants knew that one of their choices would be realized in the end, and they would have to eat whatever they chose on the trial that was randomly drawn for being paid out.

\section{Psychometric inventories}

German versions of the Spielberger State-Trait Anxiety Inventory (Laux et al., 1981), Three Factor Eating Questionnaire (Pudel and Westenhöfer, 1989), and Behavioral Inhibition and Activation Scales (Strobel et al., 2001) were administered at the end of the study. Data for the trait anxiety scale of the State-Trait Anxiety Inventory are missing for one participant, as he failed to complete the second page of the questionnaire.

\section{Statistical analyses}

All behavioral data were analyzed using either the Matlab (Release 2014b, version 8.4.0.150421, MathWorks, RRID:SCR_001622) or R (Version 3.2.1; R Core Team, RRID:SCR_001905) statistical software packages. The fMRI results were depicted using the MRIcron software package (http://www.mccauslandcenter.sc.edu/mricro/mricron/, RRID:SCR_00 2403). All correlations reported in this paper were assessed with a nonparametric bootstrap method. Two-tailed $p$ values for correlations were obtained by testing the Pearson's correlation coefficients $(r)$ against a null distribution generated from 5000 permutations of the data. The $95 \%$ confidence intervals (CIs) for the correlations were computed from 5000 bootstrapped samples of the data. The multiple regression model in Equation 2 below was fit using the "lm" function in R. To visualize the HRV by taste and health difference interactions, we plot the estimated SCS levels at specific combinations of HRV and taste or health differences from this regression model (Fig. 2b,c).

\section{Heart-rate data acquisition}

We measured baseline heart rate at rest with the Polar RS 800 CX system [for a cross-validation of this method with echocardiogram (ECG), see Quintana et al., 2012]. All measurements were collected between 1:30 P.M. and 5:00 P.M. to control for circadian rhythms (Heathers, 2014). The baseline measurement was always taken in a single session before any stress or control treatment was administered. Participants were seated in a quiet room and instructed that upon mounting the Polar watch and pressing start, they would need to sit upright and remain quiet and calm during the subsequent baseline-recording interval. A baseline recording was taken for $6 \mathrm{~min}$. The first $3 \mathrm{~min}$ of the recording were discarded from the analysis to yield a set of data that were less affected by such factors as initial motion while acclimatizing to the recording environment (Quintana et al., 2016). We focused on baseline (i.e., resting) heart-rate measures to obtain a domain-general index of HRV.

\section{Heart-rate data analyses}

HRV can be calculated in two different domains: time and frequency. The full range of measures is discussed in the guidelines by the Task Force on HRV (Camm et al., 1996). Time-domain measures have the advantage of being more robust than frequency measures. Two different timedomain measures are commonly used and both characterize the distribution of interbeat intervals, which are defined as the time between two subsequent heart beats [i.e., the difference between two $\mathrm{R}$ peaks in the ECG (Guyton and Hall, 2006), hence also called the "RR interval"; Fig. 1b]. The SD of all RR (also "NN" for "normal-to-normal") intervals (SDNN) describes the total HRV within a given period (Eq. 1). The root mean square of successive differences (RMSSD) calculated between adjacent RR intervals is more sensitive to influences of short-term regulation of the heartbeat. Here we focus on HRV at rest (i.e., in the absence of specific, discrete input stimuli), and thus take SDNN as our primary measure of variability.

We chose total HRV (measured as SDNN) as our biomarker for two reasons. First, SDNN is deemed to be the most robust measure of HRV. Among all commonly computed HRV measures, it has been reported to be least compromised by different data preprocessing pipelines, especially the application of artifact correction (Salo et al., 2001). Second, the process of dietary choice is a complex behavioral outcome that may not only depend on a capacity for effective cognitive regulation that helps to achieve self-control goals, but may also be influenced by peripheral factors (e.g., endocrine status, metabolism, and energy expenditure) that are indicative of the current state of the organism. SDNN reflects all influences on the RR interval series, while it is known to correlate highly, although not perfectly, with measures that putatively reflect phasic vagal control of cardiac variability in measures taken under sedentary resting conditions (Allen et al., 2007).

The complete recording of RR intervals for each participant was extracted using the Polar software, without any transformations of the data. Three-minute intervals of the raw data were then preprocessed with the Artiifact toolbox (Version 2.08, 64-bit; Kaufmann et al., 2011), which has a better artifact detection rate and shows fewer false detections than the commonly used Kubios HRV toolbox. To identify artifacts, the Artiifact toolbox implements the algorithm of Berntson and Stowell (1998), which aims to exclude any potential artifacts before computing the criterion for identifying true artifacts. Based on the report of Salo and colleagues (2001), who compared editing procedures for correcting single RR artifacts, the identified artifacts were deleted from the RR sequence to obtain the cleanest estimate for SDNN. On average, we corrected $2.1 \%$ (SD, $\pm 3.1 \%$ ) of the RR intervals in each sample. Apart from two datasets that had a high number of artifacts requiring correction ( 12.6 and $10.5 \%$ RR intervals removed), all other datasets had between 0 and $6 \%$ artifacts corrected (21 datasets were diagnosed as free of artifacts). As a high number of corrected artifacts might be a concern for interpreting our findings, we checked all models for robustness with regard to the number of corrected artifacts.

SDNN was calculated as follows (Eq. 1):

$$
S D N N=\sqrt{\frac{1}{N-1} \sum_{j=1}^{N}\left(R R_{j}-\overline{R R}\right)^{2}}
$$



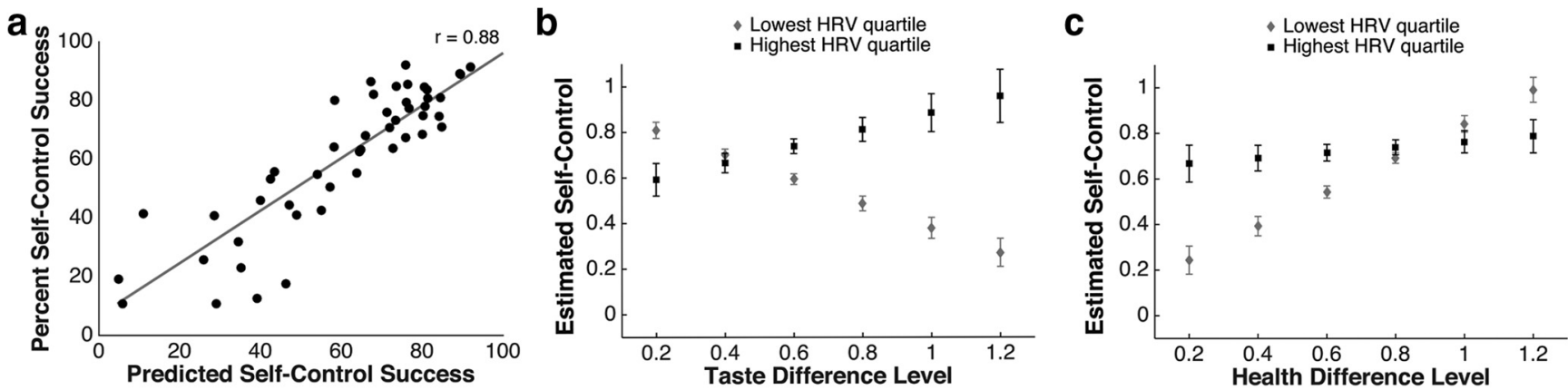

Figure 2. Combining behavioral and HRV measures to predictSCS rates. HRV and restrained eating trait (RE) were uncorrelated and predicted independent and significant portions of the variation in self-control behavior in our combined behavioral model (Table 2). $\boldsymbol{a}$, This scatter plot shows the association between predicted and observed SCS rates. We used the model listed in Equation 2 together with a LOSO procedure to predict SCS. Including the predictors for baseline HRV and its interactions with taste and health aspects yielded a coefficient of determination $R^{2}=0.77$. Moreover, omitting HRV from the predictive model resulted in a $12 \%$ decrease in explained variance. $\boldsymbol{b}, \boldsymbol{c}$, Error bar plots convey the nature of the HRV by taste and health interactions listed in Table $2 . \boldsymbol{b}$, The HRV endophenotype interacted with the level of taste temptation faced during self-control challenges. To visualize this result, the plot shows the response patterns from participants falling into the highest (black square) and lowest (gray diamond) quartiles of total HRV. The $x$-axis represents the average taste difference level (i.e., self-control difficulty) a participant faced during the dietary-choice task. The estimated SCS on the $y$-axis represents the model fit for discrete levels of the HRV by taste temptation interaction. Participants with higher HRV overcame even the largest taste temptations, whereas participants with low HRV who faced large taste temptations frequently failed to use self-control. $c$, Interaction with the average difference in health: the higher the health difference between the two foods, the more health benefit could be gained by refusing the tastier item. The $x$-axis represents the average health difference level (i.e., potential self-control benefit) a participant faced during the dietary-choice task. The estimated SCS on the $y$-axis represents the model fit for discrete levels of the HRV by health gain interaction. Participants with the lowest HRV rarely used self-control if potential health gains were small, whereas participants with the highest HRV engaged self-control at a relatively high level regardless of how much health benefit could be gained. Error bars in panels $b$ and $c$ denote standard errors of the mean for the estimated coefficients.

HRV was calculated with the Artiifact software suite, using fast Fourier transforms (Berntson and Stowell, 1998; Kaufmann et al., 2011) with an interpolation rate of $4 \mathrm{~Hz}$ (spline interpolation) and a Hanning window width that matched the total length of the edited recording $(180 \mathrm{~s}$ or slightly less in case of deletion correction). Frequency bands were bounded between 0.003 and $0.04 \mathrm{~Hz}$ for the very low frequency band, 0.04 and $0.15 \mathrm{~Hz}$ for the low-frequency band, and 0.15 and $0.4 \mathrm{~Hz}$ for the high-frequency band.

\section{fMRI data acquisition}

Images were acquired using a Philips Achieva $3 \mathrm{~T}$ whole-body scanner with an eight-channel sensitivity-encoding head coil (Philips Medical Systems) at the Laboratory for Social and Neural Systems Research, University Hospital Zurich. Stimulus presentation was controlled with the Psychophysics Toolbox Software [Psychtoolbox 3.0 (Brainard, 1997), RRID:SCR_002881]; the paradigm was presented via a back-projection system to a mirror mounted on the head coil.

We acquired gradient echo $\mathrm{T} 2{ }^{*}$-weighted echo-planar images (EPIs) with BOLD contrast (41 slices per volume; field of view, $200 \times 126.5 \times$ $200 \mathrm{~mm}$; slice thickness, $2.5 \mathrm{~mm}$; $0.6 \mathrm{~mm}$ gap; in-plane resolution, $2.5 *$ $2.5 \mathrm{~mm}$; matrix, $80 * 80$; repetition time, $2460 \mathrm{~ms}$; echo time, $30 \mathrm{~ms}$; flip angle, $77^{\circ}$ ) and a SENSE (sensitivity-encoding for fast MRI) reduction (i.e., acceleration) factor of 2 . Volumes were acquired in axial orientation at $\mathrm{a}+15^{\circ}$ tilt to the anterior commissure-posterior commissure line. We collected 161 volumes in ascending order during each of the three experimental runs, together with five "dummy" volumes at the start and end of each run. A T1-weighted turbo field echo structural image was acquired in sagittal orientation for each participant at the end of the scanning session with the same angulation that applied to the functional scans (181 slices; field of view, $256 \times 256 \times 181 \mathrm{~mm}$; slice thickness, $1 \mathrm{~mm}$; no gap; in-plane resolution, $1 * 1 \mathrm{~mm}$; matrix, $256 * 256$; repetition time, $8.4 \mathrm{~ms}$; echo time, $3.89 \mathrm{~ms}$; flip angle, $8^{\circ}$ ). To measure the homogeneity of the magnetic field, we collected B0/B1 maps before the first and second run and before acquiring the structural scan (short echo time, $4.29 \mathrm{~ms}$; long echo time, $7.4 \mathrm{~ms}$ ). We measured breathing frequency and took an ECG with the in-built system of the scanner to correct for physiological noise.

\section{fMRI preprocessing}

Functional data were spatially realigned and unwarped with statistical parametric mapping software (SPM8, Update Rev. Nr. 5236; Functional Imaging Laboratory, University College London, RRID:SCR_007037), segmented according to the participant's T1-weighted high-resolution structural image and normalized to the individual mean EPI template before smoothing with an isometric Gaussian kernel ( $4 \mathrm{~mm}$ full width at half maximum). As a last step in preprocessing, we used RETROICOR, as implemented in the PhysIO toolbox, to model respiration and heartbeat (Glover et al., 2000) to account for fluctuations in the BOLD signal due to physiological noise. The PhysIO Toolbox by Kasper et al. (2009) is distributed as open source code as part of the TAPAS (Translational Algorithms for Psychiatry-Advancing Science) software collection (www. translationalneuromodeling.org/tapas/). Following Harvey et al. (2008), the PhySIO algorithm uses Fourier expansions of different order to the estimate the phases of cardiac pulsation (third order), respiration (fourth order), and cardiorespiratory interactions (first order). For two participants, the scanner could not save physiological data due to a technical problem. For these participants, only the standard motion-correction procedure was applied.

\section{fMRI analyses}

General linear models. In all fMRI analyses, regressors in the models were defined as boxcar functions with durations equal to the reaction time on each trial. All three fMRI models also included regressors for headmotion, respiratory, and cardiac effects on each trial to account for variance in the BOLD signal associated with these sources of noise.

Our primary general linear model (GLM), GLM-CH, tested for regions that correlated with HRV during self-control challenges. The regression modeled as events of interest all trials that contained (1) a challenge, (2) no challenge, while controlling for (3) healthier experimenter recommendations and (4) less healthy experimenter recommendations. Note that the experimenter recommendations were included in the choice task to test a separate hypothesis unrelated to the current report. Those recommendations are not discussed here. Self-control challenge and no-challenge trials included parametric modulators for relative health and taste differences. We computed a first-level contrast for Challenge $>$ No Challenge trials. At the second (group) level, we examined whether increases in BOLD activity on challenge trials were correlated with HRV levels using nonparametric permutation tests $(n=$ 5000 permutations) and included threshold-free cluster enhancement (TFCE) as implemented in the function "Randomize" in the Oxford Centre for Functional MRI of the Brain (fMRIB) Software library [FSL 5, FMRIB (Winkler et al., 2014), RRID:SCR_002823]. We used FSL for the group-level analyses because the TFCE and permutation algorithms are more fully documented and run considerably faster in FSL compared with their implementation in SPM12. 
A second, separate GLM, GLM-SV, was computed to determine whether BOLD activity was related to the integrated value of the chosen food. This GLM included parametric regressors for the integrated subjective value of the chosen and nonchosen food items on every choice onset. Once again, additional regressors controlling for the impact of the experimenter recommendations were included in the model with separate regressors for events in which participants chose based on the recommendation, and in which they did not follow the recommendation. We modeled each participant's subjective value of food items on every trial by combining the weighted values for the taste and health of each food. The weights were derived from individual logistic regressions on the participant's choices (identical to Maier et al., 2015). Briefly, for each participant, a logistic regression estimated the probability of choosing the left item as a function of the taste and health of the left and right item, with all ratings $z$-scored within participant before entering them in the model. Two additional binary regressors indicated whether the left or right item had been recommended. These regressors for left and right item recommendations took the value of 1 when the item was recommended, and 0 when it was not recommended. When no recommendation was given on a trial, both regressors had a value of zero. Note that the spatial presentation of the items was completely randomized, so that the left item was equally likely to be the healthier or the tastier of both options. We took the mean of the taste betas for the left and right item obtained for each participant, averaging them into a common taste weight for this individual. The same was done for health. These averaged taste and health weights were then used to multiply the $z$-scored taste and health values of each item presented in the choice paradigm. To obtain the subjective value for each food, the weighted taste and health values were added up to a weighted subjective value separately for the left and right food items.

We computed a first-level contrast for the chosen food value for each participant and extracted betas from this contrast within our functional ROI of the vmPFC.

To examine the impact of health and taste attributes on the BOLD signal, we used a third GLM, GLM-HT, that modeled the following five events: (1) all choices, (2) trials on which the healthier food was recommended and chosen, (3) trials on which the healthier food was recommended and not chosen, (4) trials on which the less healthy food was recommended and chosen, and (5) trials on which the less healthy food was recommended and not chosen. Note that the 30 baseline trials did not contain any recommendation and, therefore, the sum of regressors $2-5$ does not equal regressor 1 . The first regressor for all choices included the following four parametric modulators: (1) health of the chosen item (Hc), (2) taste of the chosen item (Tc), (3) health of the nonchosen item (Hnc), and (4) taste of nonchosen item (Tnc). These parametric regressors were not orthogonalized with respect to one another. We computed first-level contrasts for Tc-Tnc and Hc-Hnc. We then extracted the betas for these contrasts from our functional vmPFC ROI. The significance of the correlations between BOLD sensitivity to taste and health differences in the vmPFC and HRV were determined from 5000 permutations of the data.

Anatomical masks. The combined anatomical mask for the vmPFC was constructed from a conjunction of the bilateral frontal pole, frontal medial, paracingulate, and subcallosal cortex areas that exceeded $20 \%$ probability of belonging to the respective structure in the Harvard-Oxford Cortical Atlas (HOA; Desikan et al., 2006). To limit the mask to our ROI along the medial wall, the HOA-derived anatomical mask was intersected with a rectangular box around the midline (coordinates in $\mathrm{mm}: x=$ $[-22,21], y=[-110,73], z=[-35,9])$.

The anatomical mask of the left dlPFC was constructed from a conjunction of the left inferior frontal gyrus (pars opercularis and reticularis) and left superior frontal gyrus areas that exceeded $20 \%$ probability of belonging to these structures, according to the HOA.

Because we tested two separate regions of interest (vmPFC and dlPFC), we used a critical value of $p<0.025$ (i.e., 0.05/2) for smallvolume correction at the voxel level.
Table 1. GLM predicting total HRV (GLM-HRV)

\begin{tabular}{lrrrc}
\hline Regressor & $\beta$ Estimate & \multicolumn{1}{c}{ SEM } & \multicolumn{1}{l}{$t$} & \multicolumn{1}{l}{$p$} \\
\hline Intercept & 189.41 & 31.17 & 6.08 & $<0.001$ \\
Number of artifacts & 5.07 & 1.49 & 3.41 & 0.0014 \\
Mean heart rate & -1.17 & 0.47 & -2.48 & 0.017 \\
Trait anxiety & -0.57 & 0.63 & -0.91 & 0.37 \\
\hline
\end{tabular}

Results from a GLM with possible determinants of HRV (represented as untransformed values of SDNN in milliseconds): the number of artifacts corrected in the dataset and mean heart rate (after artifact correction by deletion). To assess whether trait anxiety explains additional variance beyond an increase in mean heart rate, we added a regressor with the trait anxiety score as measured by the Spielberger State-Trait-Anxiety Inventory, so that mean heart rate and trait anxiety compete for variance in the same model. When doing so, mean heart rate accounts for a decrease in HRV, but trait anxiety does not explain further variance. The results above hold when excluding the HRV outlier from this model.

\section{Health, taste, and appetitiveness ratings}

Participants rated health, taste, and how appetizing they found the depicted foods on a continuous rating scale with anchor points from -5 for "very untasty/unhealthy" to +5 for "very tasty/healthy", or vice versa, to counterbalance order effects. Taste and health ratings were not correlated: the median correlation was $-0.09 \pm 0.31$ median absolute deviation (MAD) in the Stress group, and $-0.06 \pm 0.20 \mathrm{MAD}$ in the Control group. Neither health $(r=-0.12, p=0.40)$, nor taste $(r=0.09, p=$ $0.56)$, nor appetitiveness ratings $(r=0.13, p=0.37)$ were correlated with hunger levels.

\section{Results}

\section{HRV}

The mean duration of RR intervals across all participants was $929.3 \pm 136.3 \mathrm{~ms}$ (sample median of the median duration of RR intervals: $947 \pm 115 \mathrm{~ms}$ ), resulting in a mean heart rate of $66 \pm 10$ beats per minute in our sample (values are derived after deletion of artifacts). Our participants expressed a median total HRV (measured as SDNN) of $98.7 \pm 30.1 \mathrm{~ms} \mathrm{MAD}$ within our 3 min baseline measurement. Consistent with previous reports (Tsuji et al., 1996), HRV was inversely related to average heart rate $(\beta=$ $-1.17 \pm 0.47, T=-2.48, p=0.017$; see Table 1$)$. However, total HRV did not differ between participants later assigned to the Stress or Control groups (Stress, $98.7 \pm 29.6 \mathrm{~ms}$; Control, $97.7 \pm$ $30.9 \mathrm{~ms} ; p=0.93 ; Z=0.09$; Wilcoxon rank-sum test). Regarding biological and psychological markers of the stress reaction, baseline SDNN and cortisol reactions (area under the curve with respect to ground; Pruessner et al., 2003) were not significantly correlated $(r=-0.19, p=0.18)$, nor were baseline SDNN and perceived stress $(r=-0.10, p=0.49)$. Visual inspection of a scatter plot revealed one outlier in the SDNN measure: the value for this participant fell between 2 and 3 SDs from the sample mean. Therefore, we checked our results for robustness with and without this participant's data and found that all results remained significant in both cases.

One concern in the evaluation of HRV is that applying artifact correction might inflate indices of HRV (Heathers, 2014; Quintana and Heathers, 2014) and as few as one edited artifact in the RR interval series may do so (Berntson and Stowell, 1998). Indeed, we observed a significant positive correlation between the number of artifacts that we corrected per dataset and the SDNN $(r=0.44, p=0.003$; CI $[0.26,0.61])$. Thus, we included the number of corrected artifacts and mean heart rate as additional covariates in our regression model to test whether HRV would be predictive beyond these influences.

\section{$H R V$ and self-control behavior}

We originally defined SCS as choosing the healthier, but less tasty, of two food items in challenging trials in which health and taste conflicted, meaning that the participant had to overcome his own taste preferences to choose the healthier option. We initially 
tested the relationship between total HRV (i.e., SDNN) and SCS in a bivariate correlation analysis. Total HRV was associated with the frequency of SCS in the dietary-choice task over all participants (Pearson's $r=0.36, p=0.01$; CI $[0.07,0.59]$; all $p$ values are derived from 5000 permutations of the data; excluding the HRV outlier: $r=0.33, p=0.02$, CI $[0.03,0.58])$. For comparison, the correlation between SCS and the cognitive restraint in eating score (RE) obtained from the Three Factor Eating Questionnaire (Pudel and Westenhöfer, 1989) was $r=0.35$ ( $p=0.01$; CI [0.11, $0.55]$ ). This restraint score captures the degree to which individuals use cognitive strategies to limit calorie intake, for example by counting calories, deliberately picking small portions of food, or consuming foods with lower calorie content. Thus, as a biomarker of dietary self-control, HRV explains approximately the same amount of individual variance in choice behavior as an established psychometric index of eating behavior. Restrained eating scores were not significantly associated with HRV $(r=$ $0.14, p=0.35$; CI $[-0.28,0.42])$, suggesting that the two measures could be readily combined to explain additional variation in self-control behavior.

Therefore, we modeled SCS (i.e., choosing a healthier, less tasty item) in a multiple regression that included both HRV and RE together (Eq. 2). Beyond testing whether HRV and RE could be combined to explain additional variance in self-control, we included RE in the model because it is a widely used, validated measure for dietary SCS (Laessle et al., 1989; Allison et al., 1992; Williamson et al., 2007). As such, it serves as a benchmark for judging the utility of HRV as a biomarker for dietary self-control. When including both RE and HRV into the same model, we can assess whether HRV is predictive beyond a known trait characteristic of dietary SCS. SCS was calculated according to the following equation (Eq. 2):

$$
(\text { Stress }+\mathrm{HRV}+\mathrm{RE}) *(\text { Hdiff }+ \text { Tdiff })+\text { error. }
$$

This regression allowed us to examine potential interactions between the individual characteristics of HRV and RE and task features, such as the stress manipulation (Stress), as well as the average health (Hdiff) and taste differences (Tdiff) a participant faced within the food-choice task. Note that although all participants faced self-control challenges in the food-choice task, the degree of the challenges depended on each individual's opinions on the taste and healthiness of the various foods.

Higher HRV and RE characteristics reduced the influence of taste temptations in self-control dilemmas. All results from the regression in Equation 2 are listed in Table 2. Higher HRV levels increased the degree to which high taste temptations (i.e., taste differences) were overcome, leading to greater SCS (Fig. 2b). Moreover, unlike low-HRV participants, those with high HRV successfully used self-control regardless of the average health difference between the two options, suggesting that they engaged self-control even when the benefit of doing so (i.e., the increase in healthiness) was relatively small (Fig. 2c). A similar interaction was observed between RE and taste differences. As previously reported (Maier et al., 2015), acute stress reduced the use of selfcontrol in dietary choice. To determine whether HRV could act as a buffer against acute stress, we computed an extended version of the model in Eq. 2 that also included interactions between stress and HRV and between stress and RE. However, we did not observe significant interactions between Stress and HRV or RE, indicating that the relationship between HRV and dietary selfcontrol persisted in both in the Stress and Control groups, but that HRV was not associated with resilience to acute stress. For
Table 2. Predictors of SCS

\begin{tabular}{lrlrl}
\hline Fixed effects & $\beta$ Estimate & \multicolumn{1}{l}{ SEM } & \multicolumn{1}{l}{$T$} \\
\hline Intercept & 0.45483 & 0.04434 & 10.258 & $2.28 \mathrm{e}-12$ \\
Stress & -0.07869 & 0.06450 & -1.220 & 0.23021 \\
Tdiff & -0.02450 & 0.11721 & -0.209 & 0.83555 \\
Hdiff & 0.35598 & 0.10108 & 3.522 & 0.00116 \\
HRV & 0.01239 & 0.03785 & 0.327 & 0.74524 \\
RE & 0.02995 & 0.03951 & 0.758 & 0.45326 \\
Stress $\times$ Tdiff & -0.38367 & 0.11756 & -3.264 & 0.00237 \\
Stress $\times$ Hdiff & 0.33003 & 0.11868 & 2.781 & 0.00848 \\
HRV $\times$ Tdiff & 0.30100 & 0.06253 & 4.814 & $2.51 \mathrm{e}-05$ \\
HRV $\times$ Hdiff & -0.20929 & 0.06429 & -3.255 & 0.00242 \\
RE $\times$ Tdiff & 0.23935 & 0.07978 & 3.000 & 0.00481 \\
RE $\times$ Hdiff & -0.17495 & 0.08761 & -1.997 & 0.05323 \\
\hline
\end{tabular}

Results from a GLM of SCS. SCS was computed as the mean number of trials in which participants chose the healthier less tasty item in trials in which health and taste were not aligned (challenge trials). Resting HRV was defined as the SD of all RR intervals over a 3 min period. The cognitive restraint in eating score (RE) was obtained from the Three Factor Eating Questionnaire (Pudel and Westenhöfer, 1989). The regressor, Stress, is a binary factor indicating that the participant underwent the stress manipulation. The average health (Hdiff) and taste difference (Tdiff) regressors represent the mean taste temptation and health gain that participants were faced with during the dietary choice task. All estimates are reported with their SEM.

simplicity and ease of interpretation, we report the reduced model without interaction terms in Table 2.

As a robustness check, we controlled for the influences of the HRV outlier and several other factors that might relate to HRV. We estimated the basic model (Eq. 2) without the HRV outlier, and added the following: age, the combined number of cardio and strength exercise sessions per week, BMI, hunger level, trait anxiety score, mean heart rate, and number of corrected artifacts in the recording as nuisance regressors. The results were qualitatively unchanged and we still observed a significant relationship between taste and HRV as described above $(T=4.77, p=4.85 \mathrm{e}-$ $05)$. Note that we included trait anxiety in this robustness check because previous work has shown that trait anxiety is also correlated with HRV (Gaburro et al., 2011; Verkuil et al., 2014), and therefore we tested for this relationship in our data as well. We used the trait scale of the Spielberger State-Trait Anxiety Inventory as our measure of anxiety (median score for our sample: $34.5 \pm 5.9$ out of possible score ranging from 20 to 80 ). Consistent with previous reports, we observed a negative correlation between HRV and trait anxiety scores (permutation $r=-0.28$, $p=0.0488$; CI $[-0.48,-0.06])$. Trait anxiety scores were also positively correlated with mean resting heart rates across participants $(r=0.39, p=0.01$; CI $[0.11,0.60])$.

To assess the predictive qualities of RE and HRV with regard to self-control in a more robust way, we predicted self-control levels out-of-sample using the leave-one-subject-out (LOSO) method. After taking one participant's data out of the sample, we fit the model in Eq. 2 to explain the variance in self-control levels of the remaining participants. Using the $\beta$ coefficients from the training set, we then predicted the self-control level of the left-out participant. Squaring the obtained correlation coefficient for the true and predicted self-control levels in the full model $(r=0.88$, $p<0.0001$, CI $[0.81,0.92])$ yielded the coefficient of determination for the model with combined predictors of RE and HRV, $R^{2}=0.77$ (Fig. $2 a$ ). For comparison, fitting the model without the predictors for HRV and its interactions yielded a lower correlation between true and predicted self-control levels $(r=0.81$, $p<0.0001$, CI $[0.63,0.88])$, leading to an observed $R^{2}=0.65$. That is, by combining RE and HRV, we could significantly explain an extra $12 \%$ of the variation in out-of-sample self-control rates. Using a "split half" instead of an LOSO procedure (i.e., randomly sampling half the dataset for model fitting and using 

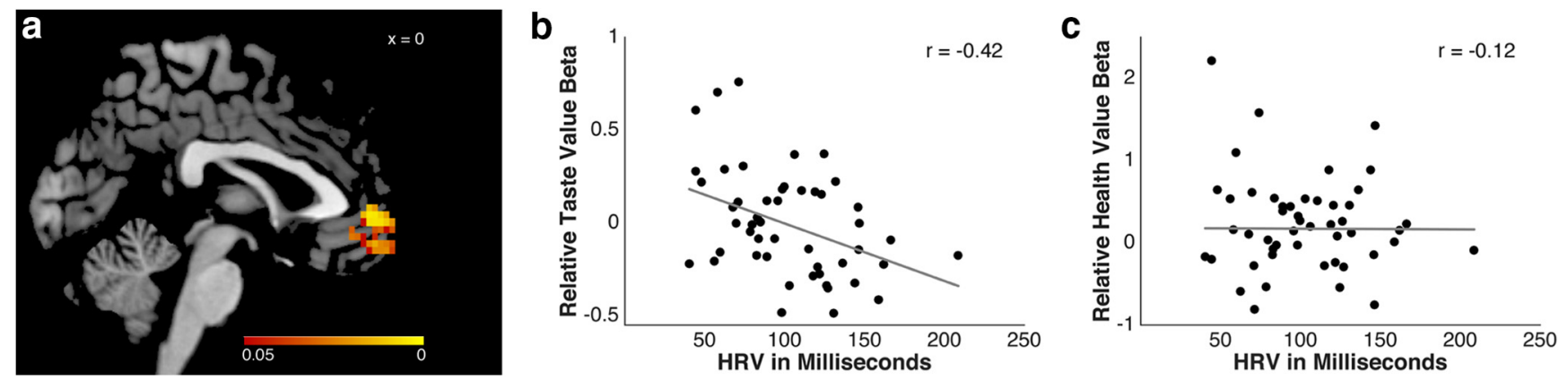

Figure 3. HRV is correlated with BOLD signal in the vmPFC. $\boldsymbol{a}$, Baseline HRV positively correlated with higher activity in Self-Control Challenge $>$ No Challenge trials in the vmPFC ( $p<0.05$, small-volume corrected). The color bar represents small-volume corrected $p$ values. $\boldsymbol{b}$, Within this functional ROI, the relative taste representation (taste of the chosen minus taste of the nonchosen food) correlated negatively with individual HRV $(r=-0.42, p=0.002)$. , There was no significant correlation between HRV and the relative health value $(r=-0.12, p=0.42)$.

the remaining half for predicting out of sample) yielded a similar benefit (16\%) for including HRV in the predictive model (full model: $r=0.81, p<0.0001$, CI $[0.55,0.92], R^{2}=0.66$; model without HRV: $r=0.71, p=0.0004$, CI $[0.26,0.89], R^{2}=0.50$ ).

During the initial review of this manuscript, an anonymous reviewer made the insightful suggestion that we could also test the relationship between HRV and another form of self-control in our dataset. Our food-choice paradigm included recommendations about which food to choose, and in some cases these recommendations were in favor of the unhealthy food (participants were told that recommendations were usually, but not always in favor of the healthier item). This feature enables us to test whether HRV is associated with the fraction of trials in which participants overrode this unhealthy recommendation and still chose the healthier food. We found that there was also a positive correlation between $\mathrm{HRV}$ and this alternative measure of control $(r=0.31, p=0.01$, CI $[0.01,0.56])$. These two measures of self-control are not independent because both are based, in part, on the incorporation of health attributes into the choice process (correlation between self-control measures: $r=0.77, p<0.0001$, CI $[0.620 .87])$. Even so, this post hoc finding is consistent with the idea that $\mathrm{HRV}$ is a domain general marker of self-regulatory ability or efficiency as outlined in the introduction section.

\section{HRV and BOLD activity during self-control}

To investigate whether HRV could serve as a biomarker of changes in the brain's decision circuitry in the food self-control paradigm, we analyzed BOLD activity measured during the choice task. Our primary general linear model (GLM-CH) tested for regions that correlated with HRV during self-control challenges.

HRV positively correlated with self-control at the level of observed choices. We hypothesized, therefore, that HRV would be associated with BOLD activity in regions known to be involved in the value computation process during self-controlled choices, namely the vmPFC and dIPFC. We tested this hypothesis in anatomical masks of the vmPFC and dlPFC based on the HarvardOxford Cortical Atlas (Desikan et al., 2006). The vmPFC mask comprised the bilateral vmPFC that is part of the brain's valuation system (Bartra et al., 2013; Clithero and Rangel, 2014; Abitbol et al., 2015; Pessiglione and Delgado, 2015) and has been shown to integrate taste and health values in the dietary selfcontrol paradigm (Hare et al., 2009, 2011, 2014; Foerde et al., 2015; Maier et al., 2015) as well as the separate characteristics of multiattribute choices in other, nonfood domains (Kahnt et al., 2011; Rudorf and Hare, 2014). Our second anatomical mask included the region of the left dlPFC that has been presumed to modulate activity in the vmPFC during self-control choices
(Hare et al., 2009, 2011, 2014). We found that BOLD activity in portions of the vmPFC increased as a function of baseline HRV in Challenge $>$ No Challenge trials [MNI peak: [ 1460 ] in the paracingulate/cingulate gyrus, small-volume-corrected (SVC) $p=0.004, T=4.8$, and a separate, more dorsal cluster in the cingulate gyrus at [21 41 9], SVC $p=0.038, T=3.86$; Fig. 3a]. However, we found no association between $\mathrm{HRV}$ and BOLD activity in the left dlPFC for the Challenge $>$ No Challenge trials that survived SVC within the anatomical region of the left dlPFC. Exploratory whole-brain analyses yielded no other regions that survived correcting for multiple comparisons. The results of these exploratory analyses can be accessed in a Neurovault repository under the following link: http://www.neurovault.org/collections/DNXFVQPJ/.

To establish that activity in this vmPFC region (the larger cluster with a peak at $x, y, z=1460$ ) was relevant to the participants' choices, we tested whether the chosen food values were represented in the functional region of interest (ROI) correlating with HRV. An integrated value of the chosen food was calculated in a separate GLM (GLM-SV) and we extracted the betas for this chosen food value in the vmPFC ROI. We found that activity in this ROI encoded the integrated value of the chosen food $\left(T_{(46)}=\right.$ 3.52; $p<0.001$ ).

Given our behavioral data linking HRV to the relative influence of taste on dietary choices, we tested whether HRV was also correlated with the degree to which BOLD activity in the vmPFC ROI represented taste attributes (Taste chosen - Nonchosen from GLM-HT). We found that HRV was negatively correlated with the relative taste value representation (Pearson's $r=-0.42$, $p=0.002$, CI $[-0.60,-0.19]$; Fig. $3 b)$, but not correlated with the relative health value $(r=-0.12, p=0.42$, CI $[-0.43,0.21]$; Fig. 3c). Excluding the HRV outlier did not change the results (taste $r=-0.43, p=0.004$, CI $[-0.63,-0.17]$; health $r=-0.09$, $p=0.56$, CI $[-0.43,0.23])$.

\section{Discussion}

We found that higher HRV is associated with better self-control in the face of dietary challenges. More specifically, our results show that the choices of individuals with higher HRV are less affected by tempting taste attributes than choices of participants with lower HRV. In parallel, at the neural level, higher HRV correlated with a decreased representation of taste attributes in the vmPFC, a brain region that has been associated with both regulating autonomic responses (Benarroch, 1993) and calculating subjective values of choice options (Bartra et al., 2013; Clithero and Rangel, 2014; Abitbol et al., 2015; Pessiglione and Delgado, 2015). HRV is a measure of physiological fitness that 
relates to the integrated functioning of the nervous and cardiac systems. Similarly, successful self-control relies on the integration, and potentially modified evaluation, of actions in the context of higher-order goal attainment. Our data indicate a significant association between these integration processes at the basic physiological (i.e., HRV) and cognitive (i.e., self-control) levels, suggesting that HRV measures may serve as a useful and readily obtainable biomarker for self-control abilities.

Resting HRV measured over a few minutes with relatively inexpensive and commercially available equipment predicted subsequent self-control in a dietary-choice task as well as a validated psychometric index of dietary behavior [restrained eating scale of the Three Factor Eating Questionnaire (RE)]. Moreover, as a physiological measure presumably outside the domain of conscious control, HRV also has the advantage of being immune to socially desirable reporting (Logan et al., 2008; DeVylder and Hilimire, 2015) or memory errors that can affect the accuracy of self-reports. However, when entered into a joint model, both HRV and RE were significantly related to dietary self-control, suggesting that they explained separate components of the variance in dietary choice. Thus, it is possible to combine biomarkers, such as HRV, with behavioral and self-report measures (e.g., the Three Factor Eating Questionnaire) to predict future self-control more accurately.

The fact that such biomarkers as HRV can be easily acquired and readily combined with other survey or task-based measures of self-control is important because, taken in isolation, any single measure is likely to reveal only a partial picture of self-control abilities or proclivities. In a recent meta-analysis by Duckworth and Kern (2011), informant-report and self-report questionnaires, behavioral readouts of executive function, and delayof-gratification measures showed only moderate convergent validity. In other words, self-control assessed in one fashion was only moderately related to self-control measured in another manner. In agreement with those authors, we believe that selfcontrol is a multidimensional construct best assessed, and potentially forecasted, by combining measures taken across multiple domains, including behavior, self and informant report, and both neural and more general physiological markers, such as HRV.

HRV can explain individual differences in self-control that are robust to changes in environmental context. We have previously shown that experiencing an acute stressor results in diminished self-control in the $45 \mathrm{~min}$ period following stressor onset (Maier et al., 2015). In the same sample, we find that resting HRV before stressor onset predicts the level of self-control following stress as well as it predicts choice in the control (i.e., not stressed) participants. Thus, the association between HRV and self-controlled behavior is maintained under the influence of acute stress, suggesting that the association between HRV and self-control may be context-independent.

Our current findings linking resting HRV to subsequent selfcontrol performance extend previous work reporting correlations between HRV and neural activity measured simultaneously during affective and cognitive tasks. In a study using positron emission tomography (PET) and an emotion task, Lane et al. (2009) measured regional cerebral blood flow (rCBF) when participants immersed themselves during 1 min blocks into positive, negative, and neutral emotions (evoked by film clips and vignettes of personal emotional memories) while parallel PET and HRV were recorded. During the presentation of emotional (vs neutral) stimuli, HRV correlated with $\mathrm{rCBF}$ in the caudate, midbrain, left insula, and medial prefrontal cortex. When excluding all emotion-specific activation, HRV correlated with $\mathrm{rCBF}$ in the right dlPFC, the bilateral parietal cortex, and the left rostral anterior cingulate cortex (ACC) with high-frequency (parasympathetic) components of HRV. Similarly, Gianaros et al. (2004) used PET to correlate HRV with changes in rCBF in medial orbitofrontal cortex (OFC), insula, ACC, amygdala, hippocampus, and cerebellum as a function of task demand in a workingmemory paradigm. A study by Nugent et al. (2011) found HRV to be correlated with $\mathrm{rCBF}$ in lateral and medial OFC when participants had to achieve different levels of strength in a handgrip task.

In contrast to Lane and colleagues' (2009) emotion task results and our a priori predictions, we did not observe any significant correlations between HRV and activity in the dlPFC during dietary choices. However, the differences in HRV indices and measurement times (resting vs task) preclude direct comparisons between the previous emotion regulation and current dietary self-control results. It is possible that HRV measures collected during the self-control task would tie in more closely with regulation processes in the dlPFC. However, our goal in the current study was to test whether simple, task-independent measures of HRV are associated with dietary self-control. What is consistent across studies is that individual differences in HRV are correlated with activity in neural regions linked to task performance across several domains (e.g., emotion regulation, working memory, physical effort, and dietary self-control). Together these results indicate that efforts to link cognition with central and peripheral neurophysiology may promote a better understanding of the nature of individual differences in health and cognitive behaviors, and provide opportunities for prediction and early intervention against dysfunctions (Sokol-Hessner et al., 2009; Raio et al., 2013).

Our study represents an important initial step in linking total HRV to self-control ability. This result suggests total HRV should be considered when investigating links between self-control and allostatic capacity in addition to more direct indices of phasic vagal cardiac control of HRV, such as RMSSD and high-frequency HRV. One rationale for doing so in the domain of dietary self-control is that frequency components outside the high-frequency spectrum may include information on metabolic and endocrine processes that are directly relevant to dietary decisions. These components contribute to the total HRV, but oscillate on a slower time scale (Berntson et al., 1997).

Further progress could be made by addressing this question with causal manipulations, for example by inducing endocrine signals of hunger and satiety and investigating whether the association between total HRV and self-control success varies during these states. Another interesting avenue to pursue is whether plasticity-induced changes that enable better regulation, for example through transcranial electrical or magnetic stimulation of the dlPFC, might also lead to an increase in HRV. A study in autistic children suggests this might be the case: Wang et al. (2016) found that weekly treatment with low-frequency repetitive transcranial magnetic stimulation for 3 months both improved chronic autonomic imbalance (i.e., higher low-frequency and lower high-frequency contributions to total HRV, putatively reflecting a tonically high arousal level due to activation of the sympathetic nervous system) and reduced the tonically elevated skin conductance levels commonly seen in autism. This change was accompanied by decreased irritability, reduced hyperactivity, and less stereotyped and compulsive behavior in the autistic children. Future work could therefore address this regulatory mechanism in a healthy population with a similar causal manipulation by stimulation techniques to further explore the nature of the link 
between neural correlates of self-regulation and physiological markers of allostatic capacity.

In conclusion, HRV is a marker of cardiovascular and mental health. Our results indicate that HRV also explains significant variation in self-control during dietary choice. Moreover, both HRV and a standard psychometric scale of restrained eating contributed independently to explaining variance in our behavioral model of self-control and could be used in combination to better predict dietary self-control levels.

\section{References}

Abitbol R, Lebreton M, Hollard G, Richmond BJ, Bouret S, Pessiglione M (2015) Neural mechanisms underlying contextual dependency of subjective values: converging evidence from monkeys and humans. J Neurosci 35:2308-2320. CrossRef Medline

Allen JJ, Chambers AS, Towers DN (2007) The many metrics of cardiac chronotropy: a pragmatic primer and a brief comparison of metrics. Biol Psychol 74:243-262. CrossRef Medline

Allison DB, Kalinsky LB, Gorman BS (1992) A comparison of the psychometric properties of three measures of dietary restraint. Psychol Assess 4:391-398. CrossRef

Bartra O, McGuire JT, Kable JW (2013) The valuation system: a coordinatebased meta-analysis of BOLD fMRI experiments examining neural correlates of subjective value. Neuroimage 76:412-427. CrossRef Medline

Beauchaine T (2001) Vagal tone, development, and gray's motivational theory: toward an integrated model of autonomic nervous system functioning in psychopathology. Dev Psychopathol 13:183-214. CrossRef Medline

Beauchaine TP, Gatzke-Kopp L, Mead HK (2007) Polyvagal theory and developmental psychopathology: emotion dysregulation and conduct problems from preschool to adolescence. Biol Psychol 74:174-184. CrossRef Medline

Benarroch EE (1993) The central autonomic network: functional organization, dysfunction, and perspective. Mayo Clin Proc 68:998-1001. Medline

Berntson GG, Stowell JR (1998) ECG artifacts and heart period variability: don't miss a beat! Psychophysiology 35:127-132. CrossRef Medline

Berntson GG, Bigger JT Jr, Eckberg DL, Grossman P, Kaufmann PG, Malik M, Nagaraja HN, Porges SW, Saul JP, Stone PH, van der Molen MW (1997) Heart rate variability: Origins, methods, and interpretive caveats. Psychophysiology 34:623-648. CrossRef Medline

Brainard DH (1997) The psychophysics toolbox. Spat Vis 10:433-436. CrossRef Medline

Brändle J, Preissl H, Draganova R, Ortiz E, Kagan KO, Abele H, Brucker SY, Kiefer-Schmidt I (2015) Heart rate variability parameters and fetal movement complement fetal behavioral states detection via magnetography to monitor neurovegetative development. Front Hum Neurosci 9:147. CrossRef Medline

Camm AJ, Malik M, Bigger JT, Breithardt G, Cerutti S, Cohen RJ, Coumel P, Fallen EL, Kennedy HL, Kleiger RE, et al. (1996) Heart rate variability: standards of measurement, physiological interpretation, and clinical use. Eur Heart J 17:354-381. CrossRef Medline

Clithero JA, Rangel A (2014) Informatic parcellation of the network involved in the computation of subjective value. Soc Cogn Affect Neurosci 9:1289-1302. CrossRef Medline

Daly M, Baumeister RF, Delaney L, MacLachlan M (2014) Self-control and its relation to emotions and psychbiology: evidence from a day reconstruction method study. J Behav Med 37:81-93. CrossRef Medline

Desikan RS, Ségonne F, Fischl B, Quinn BT, Dickerson BC, Blacker D, Buckner RL, Dale AM, Maguire RP, Hyman BT, Albert MS, Killiany RJ (2006) An automated labeling system for subdividing the human cerebral cortex on MRI scans into gyral based regions of interest. Neuroimage 31:968-980. CrossRef Medline

DeVylder JE, Hilimire MR (2015) Screening for psychotic experiences: social desirability biases in a non-clinical sample. Early Interv Psychiatry 9:331-334. CrossRef Medline

Duckworth AL (2011) The significance of self-control. Proc Natl Acad Sci U S A 108:2639-2640. CrossRef Medline

Duckworth AL, Kern ML (2011) A meta-analysis of the convergent validity of self-control measures. J Res Pers 45:259-268. CrossRef Medline

Foerde K, Steinglass JE, Shohamy D, Walsh BT (2015) Neural mechanisms supporting maladaptive food choices in anorexia nervosa. Nat Neurosci 18:1571-1573. CrossRef Medline
Gaburro S, Stiedl O, Giusti P, Sartori SB, Landgraf R, Singewald N (2011) A mouse model of high trait anxiety shows reduced heart rate variability that can be reversed by anxiolytic drug treatment. Int J Neuropsychopharmacol 14:1341-1355. CrossRef Medline

Gianaros PJ, Van Der Veen FM, Jennings JR (2004) Regional cerebral blood flow correlates with heart period and high-frequency heart period variability during working-memory tasks: implications for the cortical and subcortical regulation of cardiac autonomic activity. Psychophysiology 41:521-530. CrossRef Medline

Glover GH, Li TQ, Ress D (2000) Image-based method for retrospective correction of physiological motion effects in fMRI: RETROICOR. Magn Reson Med 44:162-167. Medline

Grossman P, Taylor EW (2007) Toward understanding respiratory sinus arrhythmia: relations to cardiac vagal tone, evolution and biobehavioral functions. Biol Psychol 74:263-285. CrossRef Medline

Guyton AC, Hall JE (2006) Textbook of medical physiology. Philadelphia: Elsevier Saunders.

Hansen AL, Johnsen BH, Sollers JJ 3rd, Stenvik K, Thayer JF (2004) Heart rate variability and its relation to prefrontal cognitive function: the effects of training and detraining. Eur J Appl Physiol 93:263-272. CrossRef Medline

Hare TA, Camerer CF, Rangel A (2009) Self-control in decision-making involves modulation of the vmPFC valuation system. Science 324:646648. CrossRef Medline

Hare TA, Malmaud J, Rangel A (2011) Focusing attention on the health aspects of foods changes value signals in vmPFC and improves dietary choice. J Neurosci 31:11077-11087. CrossRef Medline

Hare TA, Hakimi S, Rangel A (2014) Activity in dlPFC and its effective connectivity to vmPFC are associated with temporal discounting. Front Neurosci 8:50. CrossRef Medline

Harvey AK, Pattinson KT, Brooks JC, Mayhew SD, Jenkinson M, Wise RG (2008) Brainstem functional magnetic resonance imaging: disentangling signal from physiological noise. J Magn Reson Imaging 28:1337-1344. CrossRef Medline

Heathers JA (2014) Everything Hertz: methodological issues in short-term frequency-domain HRV. Front Physiol 5:177. CrossRef Medline

Heni M, Wagner R, Kullmann S, Veit R, Mat Husin H, Linder K, Benkendorff C, Peter A, Stefan N, Häring HU, Preissl H, Fritsche A (2014) Central insulin administration improves whole-body insulin sensitivity via hypothalamus and parasympathetic outputs in men. Diabetes 63:4083-4088. CrossRef Medline

Heni M, Wagner R, Kullmann S, Preissl H, Fritsche A (2015) Response to comment on Heni et al. Central insulin administration improves whole-body insulin sensitivity via hypothalamus and parasympathetic outputs in men. Diabetes 2014;63:4083-4088. Diabetes 64:e8-e9. CrossRef Medline

Kahnt T, Heinzle J, Park SQ, Haynes JD (2011) Decoding different roles for vmPFC and dlPFC in multi-attribute decision making. Neuroimage 56: 709-715. CrossRef Medline

Kasper LM, Vannesjö SJ, Hutton C, Dolan R, Weiskopf N, Stephan KE, Prüssmann KP (2009) Cardiac artefact correction for human brainstem fMRI at 7 tesla. Proc Org Hum Brain Mapp 15:395.

Kaufmann T, Sütterlin S, Schulz SM, Vögele C (2011) ARTiiFACT: a tool for heart rate artifact processing and heart rate variability analysis. Behav Res Methods 43:1161-1170. CrossRef Medline

Laessle RG, Tuschl RJ, Kotthaus BC, Pirke KM (1989) A comparison of the validity of three scales for the assessment of dietary restraint. J Abnorm Psychol 98:504-507. CrossRef Medline

Lane RD, McRae K, Reiman EM, Chen K, Ahern GL, Thayer JF (2009) Neural correlates of heart rate variability during emotion. Neuroimage 44: 213-222. CrossRef Medline

Laux L, Glanzmann P, Schaffner P, Spielberger CD (1981) Das State-TraitAngstinventar. Theoretische Grundlagen und Handanweisung. Weinheim, Germany: Beltz Test.

Logan DE, Claar RL, Scharff L (2008) Social desirability response bias and self-report of psychological distress in pediatric chronic pain patients. Pain 136:366-372. CrossRef Medline

Maier SU, Makwana AB, Hare TA (2015) Acute stress impairs self-control in goal-directed choice by altering multiple functional connections within the brain's decision circuits. Neuron 87:621-631. CrossRef Medline

Masi CM, Hawkley LC, Rickett EM, Cacioppo JT (2007) Respiratory sinus arrhythmia and diseases of aging: obesity, diabetes mellitus, and hypertension. Biol Psychol 74:212-223. CrossRef Medline 
McEwen BS, Wingfield JC (2003) The concept of allostasis in biology and biomedicine. Horm Behav 43:2-15. CrossRef Medline

Mischel W, Shoda Y, Rodriguez MI (1989) Delay of gratification in children. Science 244:933-938. CrossRef Medline

Moffitt TE, Arseneault L, Belsky D, Dickson N, Hancox RJ, Harrington H, Houts R, Poulton R, Roberts BW, Ross S, Sears MR, Thomson WM, Caspi A (2011) A gradient of childhood self-control predicts health, wealth, and public safety. Proc Natl Acad Sci U S A 108:2693-2698. CrossRef Medline

Nugent AC, Bain EE, Thayer JF, Sollers JJ 3rd, Drevets WC (2011) Heart rate variability during motor and cognitive tasks in females with major depressive disorder. Psychiatry Res 191:1-8. CrossRef Medline

Pessiglione M, Delgado MR (2015) The good, the bad and the brain: neural correlates of appetitive and aversive values underlying decision making. Curr Opin Behav Sci 5:78-84. CrossRef

Porges SW (1995) Orienting in a defensive world: mammalian modifications of our evolutionary heritage. A polyvagal theory. Psychophysiology 32:301-318. CrossRef Medline

Porges SW (2001) The polyvagal theory: phylogenetic substrates of a social nervous system. Int J Psychophysiol 42:123-146. CrossRef Medline

Porges SW, Raskin DC (1969) Respiratory and heart rate components of attention. J Exp Psychol 81:497-503. CrossRef Medline

Pruessner JC, Kirschbaum C, Meinlschmid G, Hellhammer DH (2003) Two formulas for computation of the area under the curve represent measures of total hormone concentration versus time-dependent change. Psychoneuroendocrinology 28:916-931. CrossRef Medline

Pudel V, Westenhöfer S (1989) Fragebogen zum Essverhalten (FEV): Handanweisung. Göttingen, Germany: Hogrefe.

Quintana DS, Heathers JA (2014) Considerations in the assessment of heart rate variability in biobehavioral research. Front Psychol 5:805. CrossRef Medline

Quintana DS, Heathers JA, Kemp AH (2012) On the validity of using the Polar RS800 heart rate monitor for heart rate variability research. Eur J Appl Physiol 112:4179-4180. CrossRef Medline

Quintana DS, Alvares GA, Heathers JA (2016) Guidelines for reporting articles on psychiatry and heart rate variability (GRAPH): recommendations to advance research communication. Transl Psychiatry 6:e803. CrossRef Medline

Raio CM, Orederu TA, Palazzolo L, Shurick AA, Phelps EA (2013) Cognitive emotion regulation fails the stress test. Proc Natl Acad Sci U S A 110:15139-15144. CrossRef Medline

Reynard A, Gevirtz R, Berlow R, Brown M, Boutelle K (2011) Heart rate variability as a marker of self-regulation. Appl Psychophysiol Biofeedback 36:209-215. CrossRef Medline

Rudorf S, Hare TA (2014) Interactions between dorsolateral and ventromedial prefrontal cortex underlie context-dependent stimulus valuation in goal-directed choice. J Neurosci 34:15988-15996. CrossRef Medline
Salo MA, Huikuri HV, Seppänen T (2001) Ectopic beats in heart rate variability analysis: effects of editing on time and frequency domain measures. Ann Noninvasive Electrocardiol 6:5-17. CrossRef Medline

Sokol-Hessner P, Hsu M, Curley NG, Delgado MR, Camerer CF, Phelps EA (2009) Thinking like a trader selectively reduces individuals' loss aversion. Proc Natl Acad Sci U S A 106:5035-5040. CrossRef Medline

Stalder T, Evans P, Hucklebridge F, Clow A (2011) Associations between the cortisol awakening response and heart rate variability. Psychoneuroendocrinology 36:454-462. CrossRef Medline

Strobel A, Beauducel A, Debener S, Brocke B (2001) Eine deutschsprachige Version des BIS/BAS-Fragebogens von Carver und White/A German version of Carver and White's BIS/BAS scales. Zeitschrift fuer Differentielle und Diagnostische Psychologie 22:216-227. CrossRef

Tangney JP, Baumeister RF, Boone AL (2004) High self-control predicts good adjustment, less pathology, better grades, and interpersonal success. J Pers 72:271-324. CrossRef Medline

Thayer JF, Brosschot JF (2005) Psychosomatics and psychopathology: looking up and down from the brain. Psychoneuroendocrinology 30:1050 1058. CrossRef Medline

Thayer JF, Lane RD (2000) A model of neurovisceral integration in emotion regulation and dysregulation. J Affect Disord 61:201-216. CrossRef Medline

Thayer JF, Lane RD (2009) Claude Bernard and the heart-brain connection: further elaboration of a model of neurovisceral integration. Neurosci Biobehav Rev 33:81-88. CrossRef Medline

Tsuji H, Venditti FJ Jr, Manders ES, Evans JC, Larson MG, Feldman CL, Levy D (1996) Determinants of heart rate variability. J Am Coll Cardiol 28: 1539-1546. CrossRef Medline

Verkuil B, Brosschot JF, Thayer JF (2014) Cardiac reactivity to and recovery from acute stress: temporal associations with implicit anxiety. Int J Psychophysiol 92:85-91. CrossRef Medline

Wang Y, Hensley MK, Tasman A, Sears L, Casanova MF, Sokhadze EM (2016) Heart rate variability and skin conductance during repetitive TMS course in children with autism. Appl Psychophysiol Biofeedback 41:47-60. CrossRef Medline

Weber CS, Thayer JF, Rudat M, Wirtz PH, Zimmermann-Viehoff F, Thomas A, Perschel FH, Arck PC, Deter HC (2010) Low vagal tone is associated with impaired post stress recovery of cardiovascular, endocrine, and immune markers. Eur J Appl Physiol 109:201-211. CrossRef Medline

Williamson DA, Martin CK, York-Crowe E, Anton SD, Redman LM, Han H, Ravussin E (2007) Measurement of dietary restraint: validity tests of four questionnaires. Appetite 48:183-192. CrossRef Medline

Winkler AM, Ridgway GR, Webster MA, Smith SM, Nichols TE (2014) Permutation inference for the general linear model. Neuroimage 92:381-397. CrossRef Medline 